\title{
Holistically convergent modular networks: a biological principle for recurrent network architecture
}

\author{
Matthew Cook ${ }^{*}$, Luca Gugelmann², Florian Jug², Christoph Krautz ${ }^{2}$ \\ From Nineteenth Annual Computational Neuroscience Meeting: CNS*2010 \\ San Antonio, TX, USA. 24-30 July 2010
}

The general principle of combining information processing modules in a feed-forward manner, to sequentially process input until the desired output has been generated, is well understood and widely used. In contrast, biological neural circuits often consist of recurrently connected modules, with each module's units encoding a different aspect of the modeled world [1,3]. No engineering principles for constructing circuits in this biological pattern are available.

We explore this biological approach to circuit synthesis by creating a network of interacting visual maps whose goal is to find a scene interpretation consistent with the input. We construct the network by specifying the modules and their relationships as shown in Figure 1. The network is not hierarchical, and the relationships bi-directional, creating recurrent loops. The input we use is the time derivative (not the magnitude) of the light intensity at each pixel, as provided by an adaptive neuromorphic sensor [2].

Since different consistency constraints are located in different parts of the network, information must propagate from the input to the ends of the network and back repeatedly as the network converges to a globally consistent state, a process we refer to as holistic convergence.

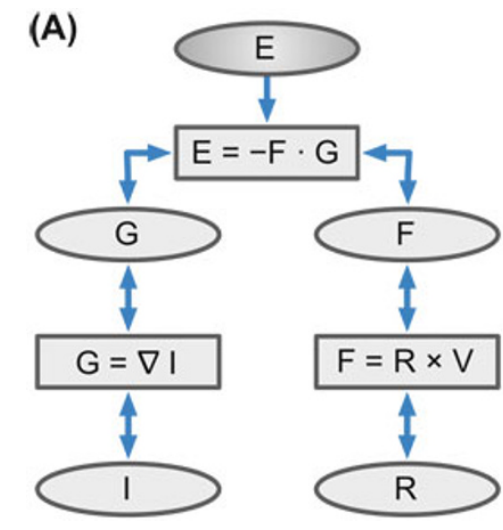

(B)

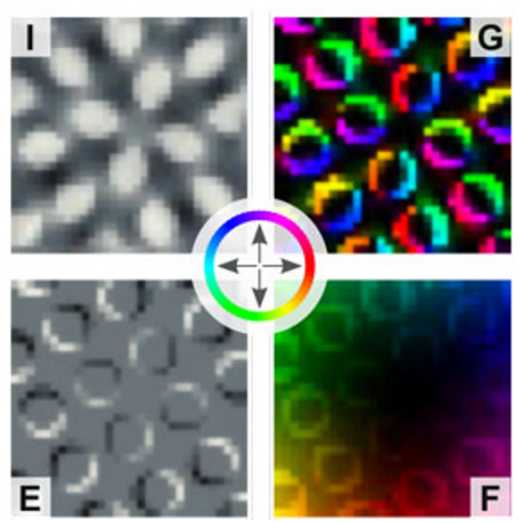

Figure 1 (A) Network architecture. $R=$ camera rotation (global 3D vector), $F=$ optic flow (2D vector map), $V=$ pixel positions projected onto sphere at focal point (constant 3D vector map), $E=$ time derivative of light intensity (scalar map), $G=$ spatial gradient of intensity (2D vector map), I = light intensity (scalar map). Each of these is a map of values covering the visual space, except for $\mathrm{R}$, which is a single global value. Note that our sensory input is E, not I. (B) Example of I, G, E, and F while the camera is rotating around its viewing axis. The circular legend indicates directional colors in $\mathrm{G}$ and $\mathrm{F}$.

* Correspondence: cook@ini.phys.ethz.ch

'Institute of Neuroinformatics, University of Zurich and ETH Zurich, Zurich, Switzerland 
Because the formula involving the input $\mathrm{E}$ leaves $\mathrm{F}$ and $\mathrm{G}$ underconstrained, one cannot directly infer $\mathrm{R}$ and I from it. Only by combining local constraints from all parts of the network can a globally coherent interpretation be obtained.

This work demonstrates how a biologically inspired recurrent architecture of modules and local relationships can be designed to interpret noisy or ambiguous data even when there is not a clear feed-forward method to generate the desired interpretation. This provides a new paradigm for the design of information processing systems, based on principles found in biological systems.

\section{Acknowledgements}

The authors would like to thank ETH Research Grant ETH-23 08-1 and EU Project Grant FET-IP-216593.

\section{Author details}

${ }^{1}$ Institute of Neuroinformatics, University of Zurich and ETH Zurich, Zurich, Switzerland. ${ }^{2}$ Institute of Theoretical Computer Science, ETH Zurich, Zurich, Switzerland.

Published: 20 July 2010

\section{References}

1. Felleman D, Van Essen DC: Distributed Hierarchical Processing in the Primate Cerebral Cortex. Cereb Cortex 1991, 1:1-47.

2. Lichtsteiner P, Posch C, Delbruck T: A 128 X 128 120db 30mw asynchronous vision sensor that responds to relative intensity change. Solid-St Circ Conf 2006, 2060-2069.

3. Ringbauer S, Bayerl P, Neumann H: Neural Mechanisms for Mid-Level Optical Flow Pattern Detection. Lect Notes Comput Sc 2007, 4669:281-290.

doi:10.1186/1471-2202-11-S1-P38

Cite this article as: Cook et al:: Holistically convergent modular networks: a biological principle for recurrent network architecture. BMC Neuroscience 2010 11(Suppl 1):P38.

\section{Submit your next manuscript to BioMed Central} and take full advantage of:

- Convenient online submission

- Thorough peer review

- No space constraints or color figure charges

- Immediate publication on acceptance

- Inclusion in PubMed, CAS, Scopus and Google Scholar

- Research which is freely available for redistribution

Submit your manuscript at www.biomedcentral.com/submit 